\title{
ATIVIDADE ANTIINFLAMATÓRIA DE CARBOIDRATO PRODUZIDO POR FERMENTAÇÃO AQUOSA DE GRÃOS DE QUEFIR
}

\author{
Maria E. C. Moreira \\ Universidade José do Rosário Vellano, Rod. MG-179, km 0, 37130-000 Alfenas - MG, Brasil \\ Marcelo H. Santos e Ivan O. Pereira* \\ Departamento de Farmácia, Universidade Federal de Alfenas, Rua Gabriel Monteiro da Silva 714, 37130-000 Alfenas - MG, \\ Brasil \\ Vany Ferraz \\ Departamento de Química, Universidade Federal de Minas Gerais, 31270-901 Belo Horizonte - MG, Brasil \\ Luiz C. A. Barbosa \\ Departamento de Química, Universidade Federal de Viçosa, 36571-000 Viçosa - MG, Brasil \\ José M. Schneedorf \\ Departamento de Ciências Exatas, Universidade Federal de Alfenas, Rua Gabriel Monteiro da Silva 714, 37130-000 Alfenas - \\ MG, Brasil \\ Recebido em 3/1/08; aceito em 30/4/08; publicado na web em 17/9/08
}

\begin{abstract}
ANTI-INFLAMMATORY ACTIVITY OF CARBOHYDRATE PRODUCED FROM AQUEOUS FERMENTATION OF KEFIR. Kefir, a symbiont microorganism suspension, presents benefic effects to health. Some kefir grains were cultivated in brown sugar, allowing to isolate a substance named CSQ. This was evaluated on a biologic essay of mouse foot edema, presenting an inhibitory activity of $30 \pm 4 \%$ against carrageenan after the stimulus. It was observed that a cultivation mean containing sucrose, and not the milky mean, lead to the production of different sugar polymeric chains of kefir. The results in vivo suggest that the CSQ exerted an anti-inflammatory activity.
\end{abstract}

Keywords: kefir; carbohydrate; anti-inflammatory.

\section{INTRODUÇÃO}

O quefir, também conhecido como kefir, é originário do eslavo Keif que significa "bem-estar" ou "bem-viver". É uma mistura probiótica original das montanhas Caucasianas da Rússia, podendo trazer vários benefícios para a saúde. ${ }^{1}$

O quefir é utilizado no Brasil como um produto da medicina popular, tendo hoje diversos efeitos probióticos relacionados à sua utilização. ${ }^{2}$ A bebida pronta para consumo contém ácido lático, assim como ácidos fórmico, succínico e propiônico, $\mathrm{CO}_{2}$, álcool etílico, diferentes aldeídos e traços de álcool isoamílico e acetona, ${ }^{3}$ além de uma variedade de folatos. ${ }^{4}$ Constitui-se de uma suspensão de microrganismos simbiontes formada por bactérias acidófilas e leveduras, que apresentam vários efeitos benéficos à saúde. ${ }^{5}$ Ele pode ser cultivado em açúcar mascavo, leite ou sucos de frutas, sendo sua coloração dependente do substrato utilizado para cultivo. Os grãos são amarelos claros quando cultivados em leite. Se crescidos em açúcar mascavo, são ocres e pardos, ou purpúreos se cultivados em suco de uva. ${ }^{6}$

Nos grãos de quefir é encontrada uma diversidade microbiológica elevada, que inclui espécies de leveduras, bactérias do ácido lático, bactérias do ácido acético e fungos. A composição da população pode diferir, dependendo da origem dos grãos ou dos diferentes métodos de cultivo. Alguns microrganismos estão sempre presentes no simbiótico, mas outros nem sempre são encontrados, dependendo do método de cultura e substrato utilizado. ${ }^{7}$ Em culturas lácteas encontra-se um polissacarídio conhecido como quefirano, ou como fator de crescimento de quefir - KGF, que constitui um bioproduto carboidratado

\footnotetext{
*e-mail: ivan.farma@bol.com.br
}

secretado por algumas bactérias presentes nos grãos de quefir e que permite o encapsulamento de suas cepas microbianas em uma matriz de polissacarídios. É um exopolissacarídio parcialmente solúvel em água, composto de unidades repetitivas de monossacarídios (galactose e glicose), na proporção de 1,1:0,9. ${ }^{5}$

Estudos realizados com quefirano demonstraram vários efeitos benéficos à saúde, dentre os quais a supressão do aumento da pressão sangüínea após 30 dias de tratamento em ratos com hipertensão induzida $;{ }^{8}$ a redução do estresse, uma vez que apresentam atividades sobre a produção de $\beta$-interferon, cortisol e noradrenalina; ${ }^{9}$ aumento da atividade fagocítica de macrófagos peritoneais e pulmonares, ${ }^{10} \mathrm{e}$ aumento de células IgA nestes sítios, ${ }^{11}$ atividade anti-tumoral $;{ }^{12}$ atividade antimicrobiana, ${ }^{13}$ efeito preventivo sobre diarréias associadas a antibióticos, por favorecer a flora intestinal normal, protegendo-a contra patógenos exógenos e mantendo seu balanço, ${ }^{1}$ aumento da atividade da dipeptidase intestinal em ratos ${ }^{14}$ redução de lipídios sanguíneos, pressão arterial, glicose sangüínea e constipação intestinal. ${ }^{15}$

Nas investigações sobre as cepas produtoras de quefirano, há controvérsias, entretanto La Rivière e colaboradores ${ }^{16}$ relataram que o Lactobacillus brevis, agora conhecido como Lactobacillus kefir, foi responsável pela produção de quefirano, ao passo que Kamdler e colaboradores ${ }^{17}$ concluíram que o L. kefir não produz o quefirano. De acordo com Toba e colaboradores, ${ }^{18}$ o principal produtor do polímero de quefirano nos grãos de quefir é o Lactobacillus kefiranofaciens e algumas outras espécies não identificadas de Lactobacillus. A produção do exopolissacarídio é uma importante característica das bactérias do ácido lático encontradas em culturas de quefir em meio lácteo. ${ }^{19}$ Será que no cultivo dos grãos utilizando açúcar mascavo como substrato é o quefirano um dos principais agentes envolvidos nas propriedades probióticas do quefir? Portanto, este estudo teve 
como objetivo isolar, caracterizar o carboidrato isolado do fermentado aquoso dos grãos de quefir cultivados em açúcar mascavo e avaliar sua atividade antiinflamatória.

\section{PARTE EXPERIMENTAL}

\section{Obtenção do carboidrato}

Cultivo dos grãos de quefir

O simbiótico utilizado foi obtido de amostras congeladas em freezer a $-50{ }^{\circ} \mathrm{C}$, presentes no Laboratório de Fitofármacos da Universidade José do Rosário Vellano - UNIFENAS, Alfenas - MG. A suspensão simbiótica de quefir foi cultivada em solução de açúcar mascavo solubilizado em água destilada $\left(50 \mathrm{~g} \mathrm{~L}^{-1}\right)$, com troca contínua do material nutriente a cada $24 \mathrm{~h}$ da cultura e sob temperatura ambiente.

\section{Reagentes}

Todos os reagentes utilizados foram de grau orgânico analítico, com exceção do açúcar mascavo comercial, obtido em fontes locais da Região dos Lagos, sul de Minas Gerais.

\section{Isolamento do carboidrato}

$\mathrm{O}$ isolamento da fração rica em carboidrato do quefir cultivado em meio aquoso foi feito de acordo com metodologia proposta por Micheli et al., ${ }^{5}$ com algumas alterações, depois de obtido o crescimento exponencial das culturas entre 7 e 15 dias. Os grãos de quefir foram colocados em um béquer contendo água destilada fervente, na proporção de 1:4, mantido sob agitação e fervura durante $1 \mathrm{~h}$. Após esse período, a mistura foi resfriada e centrifugada $\left(\right.$ Fanem ${ }^{\circledR}$, $\bmod$. 243, Brasil) a $12000 \mathrm{~g}$ por $20 \mathrm{~min}$. O precipitado foi então descartado, misturando-se o sobrenadante em igual volume de etanol $93^{\circ}$ gelado. Após esse tratamento, o produto foi mantido sob $4{ }^{\circ} \mathrm{C}$ por 15 h (overnight), sucedendo-se a centrifugação por 20 min a $12000 \mathrm{~g}$. Após a centrifugação descartou-se o sobrenadante e o precipitado foi colocado em estufa à temperatura de $50{ }^{\circ} \mathrm{C}$ por $24 \mathrm{~h}$.

\section{Caracterização da fração rica em carboidrato}

\section{Propriedades físico-químicas}

\section{Viscosidade intrínseca}

A medida de viscosidade intrínseca foi realizada a partir da medição da viscosidade relativa, $\eta_{\text {rel }}$, representada pelo quociente dos tempos de fluxo da solução polimérica e do solvente. ${ }^{20}$

Foi preparada uma solução de CSQ na concentração de 1,0 $\mathrm{mg} \mathrm{mL}^{-1}(0,1 \%)$ em água destilada Milli-Q ${ }^{\circledR}$. Após a solubilização (sob agitação, a $100{ }^{\circ} \mathrm{C}$ ) a amostra foi resfriada e a solução foi transferida para um viscosímetro analógico (Ostwald, Pyrex). O conjunto foi colocado num banho a $26^{\circ} \mathrm{C}$ por 15 min e iniciou-se a medida, por determinação do tempo de fluxo da solução polimérica (ou de eluição) numa dada região do viscosímetro. Esse procedimento foi repetido 5 vezes para cada amostra. A média dos tempos de fluxo foi dividida pelo tempo de fluxo do solvente, obtendo-se $\eta_{\text {rel }}$ Os dados foram obtidos eletronicamente pelo sistema Schott-Geraete (AVS 350).

Empregou-se a Equação de Billmeyer ${ }^{20}$ para estabelecer uma relação entre viscosidade relativa e a intrínseca,

$$
[\eta]=\frac{0,25\left\{\left(1-\eta_{r e l}\right)+3 \ln \eta_{r e l}\right\}}{c}
$$

onde $\mathrm{c}=$ concentração $(\mathrm{em} \%)$ da solução contendo o polímero.

\section{Densidade relativa}

Para a medida da densidade da solução de CSQ na concentração de $1,0 \mathrm{mg} \mathrm{mL}^{-1}\left(0,1 \%\right.$ em água Milli- $\left.{ }^{\circledR}\right)$, foi utilizado um picnômetro $\left(\right.$ Pyrex $\left.^{\circledR}\right)$ de $50 \mathrm{~mL}$, de massa conhecida $\left(\mathrm{m}_{0}\right)$. Posteriormente à introdução da amostra, adicionou-se água Milli-Q ${ }^{\circledR}$ até o picnômetro ficar totalmente preenchido. Quando o termômetro foi acoplado ao equipamento a $26{ }^{\circ} \mathrm{C}$, efetuou-se a pesagem de todo o conjunto, obtendo-se a massa da amostra com líquido $\left(\mathrm{m}_{\mathrm{SL}}\right)$. Tendo-se a massa do picnômetro cheio do líquido $\left(\mathrm{m}_{\mathrm{L}}\right)$, a massa do picnômetro contendo solução de CSQ $\left(m_{S}\right)$ e a densidade do líquido $\left(\rho_{L}\right)$, calculou-se a densidade da amostra por meio da Equação $2 .{ }^{20} \mathrm{~A}$ densidade do líquido foi calculada pelos resultados de $\mathrm{m}_{\mathrm{L}}$ dividido pelo volume do picnômetro. O procedimento foi feito em triplicata.

$\rho_{\rho}=\frac{\rho_{L}\left(M_{S}-M_{0}\right)}{\left(M_{L}-M_{O}\right)-\left(M_{S L}-M_{S}\right)}$

onde, $\mathrm{m}_{0}$ : massa do picnômetro vazio; $\mathrm{m}_{\mathrm{SL}}$ : massa da amostra com líquido; $\mathrm{m}_{\mathrm{L}}$ : massa do picnômetro cheio de líquido; $\mathrm{m}_{\mathrm{s}}$ : massa do picnômetro contendo solução de CSQ; $\rho_{\mathrm{L}}$ : densidade do líquido (a $\left.26^{\circ} \mathrm{C}\right)$.

\section{Condutividade elétrica}

Para a medida da condutividade elétrica da solução de CSQ na concentração de $1,0 \mathrm{mg} \mathrm{mL}^{-1}\left(0,1 \%\right.$ em água Milli- $\left.{ }^{\circledR}\right)$, foi utilizado um condutivímetro (Digimed $120 \mathrm{~A}$, Brasil ). O aparelho foi previamente calibrado com solução padrão de concentração $1,41 \mu \mathrm{S} \mathrm{cm}^{-1}$, sendo este padrão da marca Digimed ${ }^{\circledR}{ }^{21}$

\section{Espectrometria no infravermelho (IV)}

Para a obtenção do espectro na região do IV foi usada uma mistura com $1 \mathrm{mg}$ de CSQ pulverizado com $10 \mathrm{mg}$ de $\mathrm{KBr}$, obtendo-se uma pastilha homogênea, e seguindo-se leitura na região de 4000 a $600 \mathrm{~cm}^{-1}$, em espectrômetro de infravermelho (Perkin Elmer FTIR 1000). ${ }^{22}$

\section{Determinação de carboidratos}

Determinação de glicose livre

O teor de glicose total foi avaliado segundo método enzimático de glicose oxidase (Laborlab, Brasil). O teor foi calculado utilizando-se a Equação 3.

$C D=\frac{\left(A B S_{D}-A B S_{B}\right)}{\left(A B S_{P}-A B S_{B}\right)} \times C P$

onde, $\mathrm{CD}=$ concentração da amostra $\left(\mathrm{mg} \mathrm{dL}^{-1}\right) ; \mathrm{ABS}_{\mathrm{D}}=$ absorbância da amostra; $\mathrm{ABS}_{\mathrm{B}}=$ absorbância do branco (alíquota que não contém a amostra); $\mathrm{ABS}_{\mathrm{p}}=$ absorbância do padrão de glicose; $\mathrm{CP}=$ concentração do padrão de glicose $\left(100 \mathrm{mg} \mathrm{dL}^{-1}\right)$.

\section{Cromatografia em camada delgada (CCD)}

Uma amostra de $20 \mathrm{mg}$ de CSQ foi hidrolisada com $20 \mathrm{~mL}$ de $\mathrm{HCl}(10 \%)$, sob aquecimento com refluxo e agitação magnética por 8 h. A solução resultante foi neutralizada com carbonato de sódio e submetida à cromatografia em camada delgada em cromatoplacas de sílica gel ( 20 x $20 \mathrm{~cm}, 0,25 \mathrm{~mm}$ de espessura), juntamente com padrões de frutose, glicose, galactose, ramnose e arabinose. O sistema solvente usado foi n-butanol:ácido acético:éter etílico:água (9:6:3:1) e o revelador uma solução de anisaldeído:etanol:ácido acético:ácido sulfúrico $(0,5: 9: 0,1: 0,5)$, a $100{ }^{\circ} \mathrm{C} .{ }^{23}$ 


\section{Cromatografia gasosa}

Uma amostra de CSQ (20 mg) foi hidrolizada com $20 \mathrm{~mL}$ de $\mathrm{HCl}$ (10\%), sob aquecimento com refluxo e agitação magnética por $8 \mathrm{~h}$. Os açúcares obtidos foram convertidos em alditóis-acetatos através da reação com volumes iguais de anidrido acético e piridina $\left(120^{\circ} \mathrm{C} / 2\right.$ h), seguindo-se cromatografia gasosa (CG) das amostras. ${ }^{23}$ Utilizouse o cromatógrafo a gás (nitrogênio) Varian modelo CP3380 com coluna capilar BP20 de $25 \mathrm{~m}$ x 0,25 mm x 0,25 $\mu \mathrm{m}$. As condições estabelecidas foram temperatura do injetor $\left(250{ }^{\circ} \mathrm{C}\right)$, temperatura programada para a coluna (isoterma a $150{ }^{\circ} \mathrm{C}$ por $1 \mathrm{~min}$, e até a 240 ${ }^{\circ} \mathrm{C}$ com variação de $\left.10{ }^{\circ} \mathrm{C} \mathrm{min}^{-1}\right)$, temperatura do detector $\left(260{ }^{\circ} \mathrm{C}\right)$, e fluxo do gás de arraste $\left(2,0 \mathrm{~mL} \mathrm{~min}{ }^{-1}\right)$.

\section{Determinação da massa molecular}

A massa molecular do CSQ foi estimada através da análise por cromatografia por permeação em gel (GPC), usando-se cromatógrafo líquido Shimadzu modelo C-R4A, com coluna GPC803-D (Shimadzu) de 300 x 7,8 mm e tendo como fase móvel dimetilformamida. As condições estabelecidas foram temperatura da coluna $\left(25^{\circ} \mathrm{C}\right)$, detector de índice de refração e fluxo de $1,0 \mathrm{~mL} \mathrm{~min}^{-1}$. A amostra foi dissolvida em dimetilformamida ( $1 \mathrm{mg} \mathrm{mL}^{-1}$ ) e o volume injetado foi de $20 \mu \mathrm{L}$. Utilizaram-se padrões de poliestireno com massas moleculares de 382000, 52000, 22000 e 8500 para comparação entre os tempos de retenção destes com os da amostra de CSQ. ${ }^{24}$

\section{Atividade antiinflamatória do CSQ}

\section{Animais}

Foram utilizados ratos albinos Wistar machos $(170 \pm 50 \mathrm{~g})$, provenientes do Biotério Central da Universidade José do Rosário Vellano - UNIFENAS, Alfenas- MG, e concedidos após aprovação do trabalho pelo Comitê de Ética dessa Instituição (Protocolo 02 $\mathrm{A} / 2005$ ). Em todos os experimentos realizados in vivo, o número de ratos por grupo foi igual a 6 . Os animais foram tratados com ração comercial e água ad libitum, durante todo o experimento, garantida sua adaptação por 7 dias em sala climatizada a $23 \pm 2{ }^{\circ} \mathrm{C}$, com ciclo claro-escuro de $12 \mathrm{~h}$, e em caixas de polipropileno adequadas à sua manutenção. Ao término dos experimentos, os animais foram sacrificados por inalação de dióxido de carbono em câmaras de acrílico adaptadas.

\section{Ensaio de edema de pata induzido por carragenina}

Os animais foram subdivididos em 3 grupos ( $n=6 /$ grupo). Conforme método descrito por Winter e colaboradores, ${ }^{25} 24 \mathrm{~h}$ antes do experimento os animais ficaram em jejum. Os animais foram tratados por via oral com $1 \mathrm{~mL}$ de CSQ a $20 \mathrm{mg} \mathrm{kg}^{-1}$ (grupo 1). Os animais do grupo 2 foram tratados com $1 \mathrm{~mL}$ contendo $10 \mathrm{mg} \mathrm{kg}^{-1}$ de indometacina, e os do grupo 3, com igual volume de $\mathrm{NaCl}$ a $0,9 \%$. Após 30 min os animais foram estimulados na pata direita $(0,1 \mathrm{~mL} /$ pata $)$ com $1 \mathrm{mg} /$ pata de carragenina (Iota-Fluka Biochemika, EUA) e na pata esquerda, com igual volume de $\mathrm{NaCl}$ a $0,9 \%$, como controle.

O edema produzido nas patas de cada animal foi determinado pela diferença entre as medidas das patas esquerda e direita, utilizando um paquímetro analógico (Vernier Caliper). As patas foram aferidas antes da aplicação da substância pró-inflamatória, e de hora em hora até completar 5 h após a aplicação do estímulo.

\section{Análise estatística}

Os ensaios foram conduzidos por delineamento inteiramente casualizado, tendo como parcela um animal. Foi conduzida análise de variância dos dados expressos por média \pm erro padrão da média, satisfeitas as premissas do método, seguido por teste de Tukey-
Kramer para comparações múltiplas. Foi aceito como significativo o resultado que apresentou o P-valor menor que 0,05 em todos os cálculos. Foi utilizado pacote estatístico Origin ${ }^{\circledR}$, versão 7.5 (Microcal Origin $^{\circledR}, 7.5$, EUA), para os cálculos de ajuste não linear dos dados, e GraphPad Instat ${ }^{\circledR}$ (versão 3.01, EUA), para os cálculos de estatística inferencial.

\section{RESULTADOS E DISCUSSÃO}

As amostras suspendidas, colocadas em ebulição por $1 \mathrm{~h}$, precipitadas em solução alcoólica a 93\%, apresentaram um rendimento de $1,1 \mathrm{~g} \mathrm{~kg}^{-1}$ de grãos de quefir utilizado. Este dado foi diferente do encontrado por Micheli et al. ${ }^{5}$ e Maeda et al., ${ }^{8}$ que obtiveram um rendimento de 2 e $2,5 \mathrm{~g} \mathrm{~L}^{-1}$, respectivamente, quando isolaram o quefirano de cultura de láctea, e similar ao resultado encontrado por Rimada e Abraham, ${ }^{26}$ que isolaram o polissacarídio dos grãos de quefir cultivados em trigo $\left(1,03 \mathrm{~g} \mathrm{~kg}^{-1}\right)$.

A viscosidade intrínseca da solução de CSQ a $0,1 \%$ foi de 0,297 $\pm 0,030 \mathrm{dL} \mathrm{g}^{-1}$, sendo que o valor da solução de quefirano obtido por Micheli et al. ${ }^{5}$ foi de $5,68 \pm 0,50 \mathrm{dL} \mathrm{g}^{-1}$, em cultura de grãos de quefir em meio lácteo. Observa-se que o valor obtido para CSQ é cerca de 19 vezes menor que o valor obtido por Micheli et al. ${ }^{5}$ para o quefirano. Correlacionando a massa molecular e a viscosidade intrínseca, ${ }^{20}$ nota-se que a cadeia polimérica encontrada foi bem menor que a do quefirano obtido por culturas lácteas de quefir.

A condutividade elétrica da solução de CSQ a $0,1 \%$ foi de 2,46 $\mu \mathrm{S} \mathrm{cm}^{-1}$ sugerindo um grau de pureza similar ao valor de $1,20 \mu \mathrm{S} \mathrm{cm}^{-1}$ obtido por Micheli et al., ${ }^{5}$ que utilizaram um procedimento adicional de diálise para a obtenção do carboidrato. $\mathrm{O}$ valor da densidade relativa da solução de CSQ a $0,1 \%$ foi de $1,0443 \mathrm{~g} \mathrm{~mL}^{-1}$.

$\mathrm{Na}$ caracterização da estrutura da amostra obtida pelo espectro na região do infravermelho (IV), a substância CSQ apresentou bandas de absorção fortes entre 3600-3100 (v O-H) e $1070 \mathrm{~cm}^{-1}$ (v C-O), sugerindo presença de um componente poliidroxilado, além de bandas fracas de absorção em 2950-2880 (v C-H), 1470 e $1390 \mathrm{~cm}^{-1}\left(\delta_{\mathrm{s}} \mathrm{C}-\mathrm{H}\right)$, que caracterizam a natureza alifática dessa substância. Estes dados estão de acordo com de outros carboidratos descritos na literatura, sejam estruturas de mono, oligo ou polissacarídios. Dessa forma, compostos de estruturas diferentes podem apresentar espectros muito similares, não servindo o espectro no IV como o único parâmetro para caracterização estrutural de CSQ, mas para caracterizá-lo como um sacarídio poliidroxilado. ${ }^{22}$

Não foram detectadas quantidades significativas de glicose livre nas amostras, as quais apresentaram valores de 2,58 $\pm 0,45$ $\mathrm{mg} \mathrm{dL}^{-1}$.

Pela análise de cromatoplaca de sílica gel revelada, contendo a amostra hidrolisada e os padrões glicose, frutose, galactose, arabinose e ramnose, pôde-se constatar pelos valores próximos de Rfs $(0,41 ; 0,41 ; 0,43 ; 0,45$ e 0,55 , respectivamente), e pela coloração das manchas, a possibilidade da presença dos monossacarídios glicose, frutose, ramnose, galactose e/ou arabinose. Apesar desse método ser rápido e bastante eficaz, a determinação qualitativa para muitos compostos, neste caso, apresentou dúvidas na distinção dos monossacarídios que possuem valores de Rfs próximos. Dessa forma, utilizou-se um método mais preciso, a cromatografia gasosa (CG), que possui maior resolução para distinguir os constituintes das amostras de CSQ hidrolisada.

A análise por CG de CSQ hidrolisado confirmou a presença dos monossacarídios: glicose (40\%), ramnose (24\%), galactose (10\%) e arabinose (26\%) (Figura 1). Esse resultado difere dos trabalhos de Maeda et al. ${ }^{8}$ Frengova et al.,${ }^{19}$ Yang et al.,${ }^{27}$ Micheli et al.,${ }^{5}$ Mukai et al. ${ }^{28}$ e Kooiman, ${ }^{29}$ onde se utilizou leite como substrato de cultivo, os quais encontraram glicose e galactose como monossacarídios 


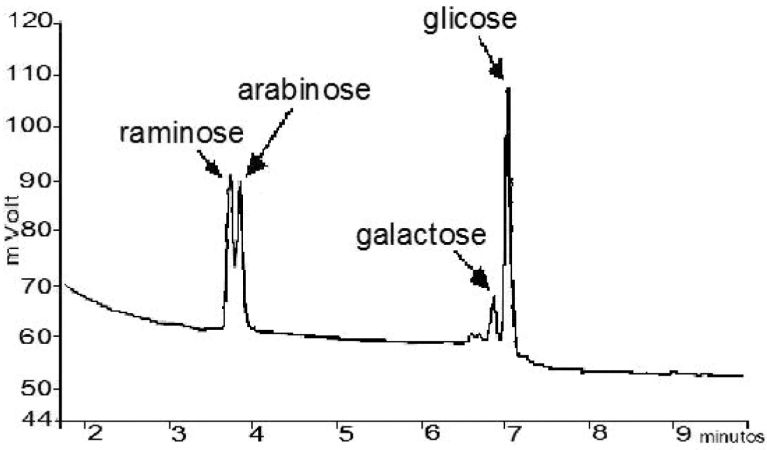

Figura 1. Cromatograma dos alditóis-acetatos do hidrolizado de CSQ, obtido por cromatografia gasosa

principais de composição da matriz polimérica.

A massa molecular do CSQ estimada por cromatografia por permeação em gel (GPC) está mostrada nas Figuras 2 e 3. Após ser estabelecida a relação entre Log (MM) e tempo de retenção para os padrões de polietileno (pela equação de regressão: $\log M M=-0,33972$ $+7,384)$, utilizaram-se os tempos dos componentes da amostra de CSQ (Figura 4) para determinar suas massas moleculares.

Pode-se verificar que foram obtidas três substâncias presentes no produto CSQ, uma das quais é a glicose. Os outros dois sacarídios parecem possuir massa molecular média de 940 e 3524 Da, que corresponde a 5 e 20 unidades de glicose, respectivamente. Como pode ser observado, os tamanhos das cadeias poliméricas encontradas no CSQ (940 e 3524 Da) são menores que a do quefirano, 760000 e $20600 \mathrm{Da}$ [ ${ }^{29,15}$ respectivamente], sugerindo tratar-se de dois componentes principais, a saber, um oligo e um polissacarídio (Figura 4). A IUPAC (International Union of Pure and Applied Chemistry - recomendação de 1996) $)^{30}$ não delimita o número de resíduos de monossacarídios presentes para ser classificado como oligo ou polissacarídio, ${ }^{30}$ mas convencionalmente é definido como oligossacarídio aquele composto que fornecerá, quando hidrolisado, 3 a 10 unidades de monossacarídios..$^{31}$ Dessa forma, podemos sugerir

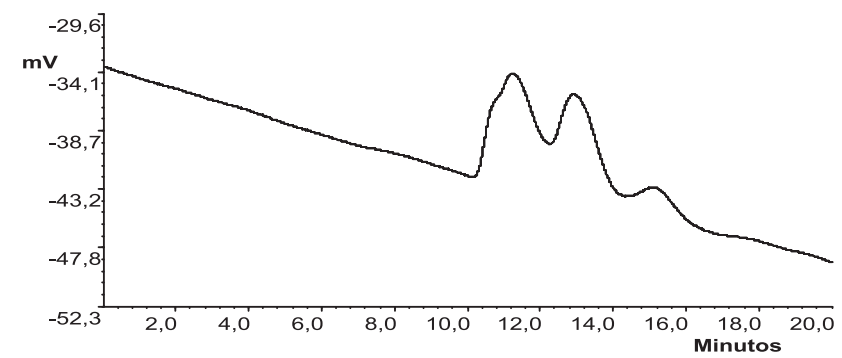

Figura 2. Cromatograma de CSQ obtido por cromatografia por permeação em gel (GPC)

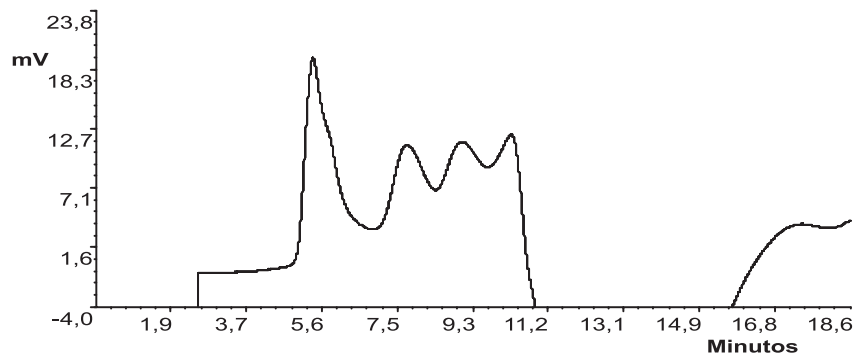

Figura 3. Cromatograma de padrões de poliestirenos, obtido por cromatografia por permeação em gel (GPC)

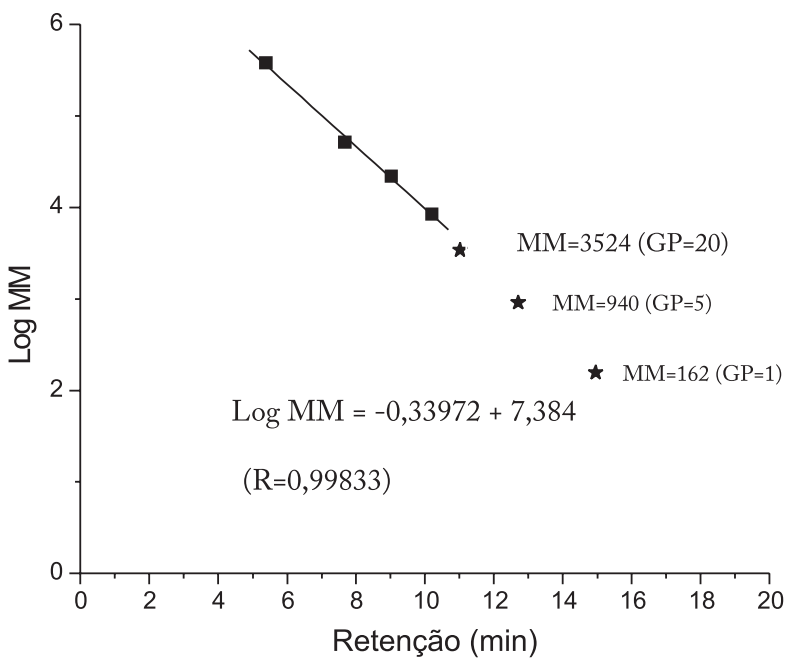

Figura 4. Massas moleculares (MM) dos padrões de poliestireno e de CSQ usando a relação logarítmica de MM e tempo de retenção das amostras por cromatografia de permeação em gel (equação de regressão: $\log M M=$ $-0,33972+7,384(R=0,99833))$. GP representa grau de polimerização da fração isolada, $\star$ amostra e $\mathbf{D a d r o ̃ e s ~ d e ~ p o l i e s t i r e n o ~}$

que o componente de maior massa se refere a um polissacarídio com 20 unidades glicosídicas; o outro composto é um oligossacarídio com apenas 5 resíduos de monossacarídios em sua constituição.

O modelo de edema de pata é o mais utilizado para se avaliar o efeito antiinflamatório de fármacos e insumos farmacêuticos. A carragenina é um polissacarídio extraído de algas que induz resposta inflamatória local mensurável. Apresenta duas fases inflamatórias e uma terceira não característica. $\mathrm{Na} 1^{\mathrm{a}}$ hora, logo após injeção da carragenina, há aumento da permeabilidade vascular mediada por histamina e serotonina. $\mathrm{Na} 2^{\text {a }}$ hora, o aumento da permeabilidade é resultado da liberação de cininas. Na $3^{\text {a }}$ hora, o aumento da permeabilidade vascular ocorre devido à ação das prostaglandinas. ${ }^{32} \mathrm{~A}$ administração de carragenina $(1 \mathrm{mg} /$ pata a $0,1 \mathrm{~mL})$ nas patas de ratos induziu edema de forma gradual (Figura 5).

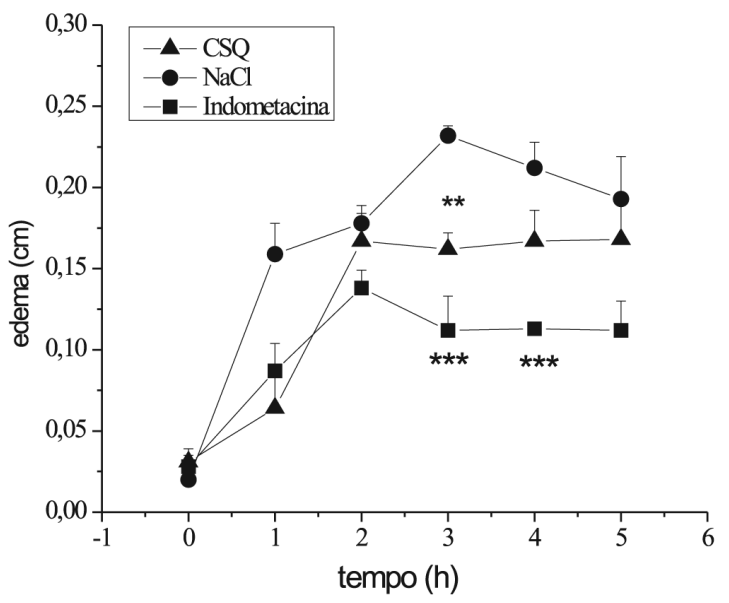

Figura 5. Edema de pata induzido por $1 \mathrm{mg}(0,1 \mathrm{~mL})$ de carragenina. Grupo controle negativo tratado com $1 \mathrm{~mL}$ de $\mathrm{NaCl}$ representados por (•); Grupo controle positivo tratado com $1 \mathrm{~mL}$ contendo $10 \mathrm{mg} \mathrm{kg}^{-1}$ de Indometacina (•);

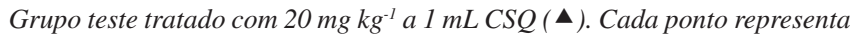
média \pm EPM (erro padrão da média) de $n=6$ por grupo. $* * * p<0,001 ;$ teste de Tukey-Kram 
O resultado mostra que o CSQ foi capaz de inibir o processo inflamatório $30 \pm 4 \%$ na $3^{\text {a }}$ hora ( $\mathrm{p}<0,001$, Tukey-Kramer). A indometacina (controle positivo) foi capaz de inibir o processo inflamatório em $52 \pm 9 \%, 47 \pm 3 \%$ na $3^{\text {a }}$ e $4^{\text {a }}$ horas, respectivamente $(\mathrm{p}<0,001$, Tukey-Kramer).

A resposta edematogênica é um dos sinais da resposta inflamatória decorrente do aumento da permeabilidade vascular, que ocorre na microcirculação, devido à ação dos mediadores liberados. ${ }^{33} \mathrm{~A}$ inibição do edema nos animais que receberam tratamento com CSQ na dosagem de $20 \mathrm{mg} \mathrm{kg}^{-1}$ (30 min antes da aplicação do estímulo edematogênico - carragenina), ocorreu na $3^{\text {a }}$ hora, sendo similar à do grupo tratado com indometacina, o que sugere um mecanismo de ação baseado no envolvimento de metabólitos do ácido araquidônico. ${ }^{34}$ Diante de todas estas observações, pode-se supor como um dos mecanismos de ação sistêmica do composto a inibição da síntese de prostaglandinas, o que pode ser verificado pela presença, no carboidrato, de constituintes com ação anti-edematogênica. Resultado similar a este foi relatado por Rodrigues e colaboradores ${ }^{35}$ que observaram, ao utilizar esse mesmo estímulo, uma inibição de 62 e $40 \%$ quando os animais foram tratados com suspensão de grãos de quefir e grãos desintegrados, respectivamente.

\section{CONCLUSÃO}

Os estudos realizados indicaram que a utilização de um meio de cultura contendo sacarose, e não o meio lácteo, levou à produção de cadeias poliméricas de açúcares com MM determinados por permeação em gel de 940 e 3524 Da. A natureza e a proporção dos monossacarídios identificados por CG foi de glicose, ramnose, galactose e arabinose $(4: 2,4: 1: 2,6)$, distintas das relatadas na literatura.

Finalmente, o carboidrato isolado na cultura de quefir em meio aquoso demonstrou atividade antiinflamatória, quando testado em animais em ensaio de edema de pata induzido por carragenina, em até $30 \pm 4 \%$, o que sugere sua utilização como antiinflamatório, abrindo novas perspectivas na aplicação de novos agentes antiinflamatórios oriundos de carboidratos poliméricos.

\section{MATERIAL SUPLEMENTAR}

Está disponibilizado em http://quimicanova.sbq.org.br, na forma de arquivo PDF, com acesso gratuito.

\section{AGRADECIMENTOS}

Ao apoio financeiro do Conselho Nacional de Desenvolvimento Científico e Tecnológico - CNPq, à Fundação de Amparo à Pesquisa do Estado de Minas Gerais - FAPEMIG.

\section{REFERÊNCIAS}

1. Zubillaga, M.; Weill, R.; Postaire, E.; Goldman, C.; Caro, R.; Nutr. Res. 2001, 21, 569.

2. Schneedorf, J. M.; Anfiteatro, D.; Quefir, um probiótico produzido por microorganismos encapsulados e inflamação, Tecmedd: São Paulo, 2004.

3. Guven, A.; Gulmez, M.; J. Vet. Med. 2003, 50, 412.

4. Patring, J. D. M.; Hjortmo, S. B.; Jastrebova, J. A; Svensson, U. K.; Andlid, T. A.; J'agerstad, I. M.; Eur. Food Res. Technol. 2006, 223, 633.

5. Micheli, L; Uccelletti, D.; Palleschi, C.; Crescenzi, V.; Appl. Microbiol. Biotechnol. 1999, 53, 69.

6. Guzel-Seydim, Z.; Seydim, A. C.; Greene, A. K.; J. Dairy Sci. 2000, 83, 275.
7. Garrote, G. L.; Abraham, A. G.; Antoni, G. L.; J. Dairy Res. 2001, 68, 639; Witthuhn, R. C.; Schoeman, T.; Britz, T. J.; Int. Dairy J. 2004, 15, 383.

8. Maeda, H.; Zhu, X.; Omura, K.; Suzuki, S.; Kitamura, S.; J. Agric. Food Chem. 2004a, 52, 5533.

9. Kabayama, S.; Osada, K.; Tachibana, H.; Katakura, Y.; Shirahata, S.; Cytotechnology 1997, 23, 119.

10. Viderola, C. G.; Duarte, J.; Thangavel, D.; Perdigon, G.; Farnworth, E.; Matar, C.; Eur. J. Inflammation 2005, 3, 63; Viderola, C. G.; Perdigon, G.; Duarte, J.; Farnworth, E.; Matar, C.; J. Dairy Res. 2006, 73, 472.

11. Viderola, C. G.; Perdigon, G.; Duarte, J.; Thangavel, D.; Farnworth, E.; Matar, C.; Immunobiology 2006, 211, 149.

12. Liu, J. R.; Wang, S. Y.; Lin, Y. Y.; Lin, C. W.; Nutr. and Cancer 2002, 44, 183; Murofushi, M.; Shiomi, M.; Aibara, K.; Japanese J. Med. Science Biology 1983, 36, 49; Shiomi, M.; Sasaki, K.; Murofushi, M.; Aibara, K.; Japanese J. Med. Science Biology 1982, 35, 75.

13. Rodrigues, K. L.; Caputo, L. R. G.; Carvalho, J. C. T.; Evangelista, J.; Schneedorf, J. M.; Int. J. Antimicrob. Agents 2005, 25, 404.

14. Urdaneta, E.; Barrenetxe, J.; Aranguren, P.; Irigoyen, A.; Marzo, F.; Ibáñez, F. C.; Nutr. Res. 2007, 27, 635.

15. Maeda, H.; Zhu, X.; Omura, K.; Suzuki, S.; Kitamura, S.; BioFactors 2004b, 22, 197.

16. La Rivière, J. W. M.; Kooiman, P.; Schmidt, K.; Arch. Microbiol. 1967, 59, 269.

17. Kamdler, O.; Kunath, P.; Syst. Appl. Microbiol. 1983, 4, 286.

18. Toba, T.; Arhara, K.; Adachi, S.; Milhwissenschaft 1987, 42, 565.

19. Frengova, G. I.; Simova, E. D.; Beshkova, D. M.; Simov, Z. I.; Z. Naturforsch 2002, 57, 805.

20. Mancini, S. D.; Matos, I. G.; Almeida, R. F.; Polímeros 2004, 14, 2.

21. Harris, D.; Análise Química Quantitativa, $5^{\text {a }}$ ed., LTC - Livros Técnicos e Científicos Editora S.A.: Rio de Janeiro, 2001.

22. Silverstein, R. M.; Webster, F. X.; Identificação Espectrométrica de Compostos Orgânicos, $6^{\mathrm{a}}$ ed., LTC - Livros Técnicos e Científicos Editora S.A.: Rio de Janeiro, 2000.

23. Ikan, R.; Natural products: a laboratory guide, $2^{\text {nd }}$ ed., Academic Press: California, 1991.

24. Collins, C. H.; Braga, G. L.; Bonato P. S.; Introdução a Métodos Cromatográficos, 6h ed., Editora Unicamp: Campinas, 1995.

25. Winter, C. A.; Risley, E. A.; Nuss, G. W.; Proc. Soc. Exp. Biol. Med. 1962, 111, 544.

26. Rimada, P. S.; Abraham, A. G.; J. Dairy Res 2001, 68, 653.

27. Yang, Z. N.; Staaf, M.; Huttunen, E.; Widmalm, G.; Carbohydr. Res. 2000, 329, 465.

28. Mukai, T.; Toba, T.; Itoh, T.; Adachi, S.; Carbohydr. Res. 1990, 204, 278.

29. Kooiman, P.; Carbohydr. Res. 1968, 7, 200.

30. http://www.chem.qmul.ac.uk/iupac/2carb/00n01.html\#01, acessada em Janeiro 2006.

31. El Khadem, H. S.; Carbohydrate Chemistry. Monosaccharides and their oligomers, Academic Press: California, 1988; Harborne, J. B.; Phytochemical Methods. A guide to modern techniques of plant analysis, $3^{\text {rd }}$ ed., Chapman \& Hall: London, 1998; Mahan, L. K.; Escott-Stump, S.; Krause: Alimentos, Nutrição e Dietoterapia, $11^{\mathrm{a}}$ ed., Roca: São Paulo, 2005.

32. Di Rosa, M.; Giroud, J. P.; Willoughby, D. A.; J. Pathol. 1971, 104, 15.

33. Rang, H. P.; Dale, M. M.; Ritter, J. M.; Moore, P. K.; Farmacologia, $5^{\text {a }}$ ed., Elsevier: Rio de Janeiro, 2004.

34. Carvalho, J. C. T.; Fitoterápicos anti-inflamatórios: Aspectos químicos, farmacológicos e aplicações terapêuticas, Tecmedd: São Paulo, 2004.

35. Rodrigues, K. L; Carvalho, J. C. T.; Schneedorf, J. M.; Inflammopharmacology 2005, 13, 485. 


\section{ATIVIDADE ANTIINFLAMATÓRIA DE CARBOIDRATO PRODUZIDO POR FERMENTAÇÃO AQUOSA DE GRÃOS DE QUEFIR}

Maria E. C. Moreira

Universidade José do Rosário Vellano, Rod. MG-179, km 0, 37130-000 Alfenas - MG, Brasil

Marcelo H. Santos e Ivan O. Pereira*

Departamento de Farmácia, Universidade Federal de Alfenas, Rua Gabriel Monteiro da Silva 714, 37130-000 Alfenas - MG, Brasil

Vany Ferraz

Departamento de Química, Universidade Federal de Minas Gerais, 31270-901 Belo Horizonte - MG, Brasil

Luiz C. A. Barbosa

Departamento de Química, Universidade Federal de Viçosa, 36571-000 Viçosa - MG, Brasil

José M. Schneedorf

Departamento de Ciências Exatas, Universidade Federal de Alfenas, Rua Gabriel Monteiro da Silva 714, 37130-000 Alfenas MG, Brasil

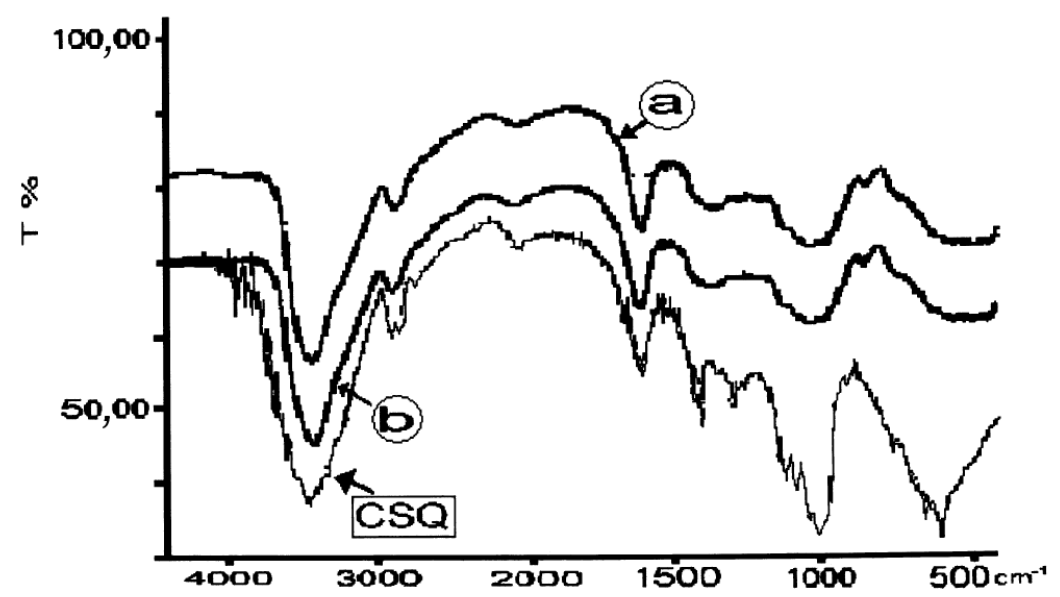

Figura 1S. Espectro no infravermelho de quefirano (a), polissacarídio LM-17 (b) e CSQ 